\title{
The diagnostic gap: Characterising the profile of undiagnosed infectious tuberculosis patients in the community
}

\author{
G Calligaro, ${ }^{1,2}$ MB BCh, FCP, MMed, Cert Pulm; A Esmail, ${ }^{1,2}$ MD, FCP, Cert Pulm; T Mnguni, ${ }^{1}$ MB ChB, FCP, MMed; \\ L Mottay, ${ }^{1} \mathrm{MB}$ BCh, FCP, Cert Pulm; K Dheda, ${ }^{1} \mathrm{MB}$ BCh, FCP, FCCP, FRCP, PhD \\ ${ }^{1}$ Division of Pulmonology, Department of Medicine, University of Cape Town, South Africa \\ ${ }^{2}$ Lung Infection and Immunity Unit, University of Cape Town Lung Institute, Groote Schuur Hospital, Cape Town, South Africa \\ Corresponding author: KDheda (keertan.dheda@uct.ac.za)
}

\begin{abstract}
Background. In tuberculosis (TB)-endemic countries, about half the total TB caseload remains undiagnosed within the community. The proportion of such patients that can potentially transmit the disease has been poorly characterised, and there is insufficient data to inform on strategies to target potentially infectious TB cases in the community.

Objective. To characterise the nature and profile of smear-positive patients diagnosed with TB in the community.

Methods. We analysed data from culture-positive TB cases in the community during the course of an intensified case finding (ICF) study. The parent study was a randomised controlled trial comparing conventional and novel diagnostics for ICF in communities in Cape Town, South Africa and Harare, Zimbabwe, where trained healthcare workers screened patients at transmission hotspots. The results of the parent study are reported elsewhere.

Results. A total of 2261 persons were screened and 875 (39\%) met the criteria for diagnostic testing. A total of 53/630 (8.4\%) had confirmed tuberculosis. Smear microscopy detected 22/53 (42\%) of the culture-positive patients. The specificity, positive predictive value (PPV) and negative predictive value (NPV) for smear microscopy were $98.4 \%, 70.0 \%$ and $95.3 \%$, respectively. No clinical or demographic variable predicted smear positivity. Only decreased culture time-to-positivity was associated with smear grade (odds ratio $0.93,95 \%$ confidence interval $0.91-0.96 ; p<0.001$ ). Conclusion. A considerable proportion of patients with undiagnosed TB in the community (almost half) were smear positive and hence potentially infectious. Interestingly, neither HIV status nor symptoms identified those patients who were potentially infectious, despite them having a higher mycobacterial burden.
\end{abstract}

S Afr Respir J 2016;22(4):93-98. DOI:10.7196/SARJ.2016.v22i4.100

Tuberculosis (TB) remains one of the world's most devastating infectious diseases, with an estimated 9.6 million incident cases globally in $2014 .{ }^{[1]} \mathrm{A}$ major problem hampering control efforts and driving the TB epidemic is the large reservoir of undiagnosed TB disease in the community, which may comprise $\sim 30$ to $50 \%$ of the total TB burden. ${ }^{[2]}$ Particularly in highburden settings, a large number of people in the community do not access healthcare or do so late in the course of the disease, and are responsible for ongoing disease transmission. Intensified case finding (ICF) seeks to address this problem by screening targeted populations for active pulmonary disease. ${ }^{[2]}$ Historically, households have been deemed to be a major focus of Mycobacterium tuberculosis transmission, but molecular epidemiological studies from sub-Saharan Africa have pointed to high transmission risk also occurring outside the home in indoor congregate settings such as the workplace or schools, or on public transport. ${ }^{[3-5]}$

Much of our current understanding of the transmissibility of pulmonary tuberculosis derives from early published reports on various community outbreaks. ${ }^{[6-10]}$ These studies have demonstrated repeatedly that a single variable - smear status - strongly predicts which patients are the most contagious. Smear-positive persons expectorate $10^{8}$ $10^{10}$ bacilli daily, or about $10^{6}-10^{7}$ per millilitre $(\mathrm{mL})$ of sputum, while smear-negative sputum contains $<10^{3}$ bacilli per $\mathrm{mL}$. ${ }^{[1]}$

Despite the contribution of undiagnosed TB in the community to the destabilisation of TB control, and the importance of undetected smearpositive cases in transmission dynamics, little is known about these potentially infectious cases. An ICF study recently performed in the impoverished communities of Cape Town, South Africa (SA), found the incidence of smear-positive disease to be $36 \%$, but the characteristics of these patients were not described. ${ }^{[12]}$ Because of this knowledge gap, we set out to characterise the nature and profile of smear-positive patients with TB, identified as part of an ICF strategy in the community.

This study was nested within a larger two-country parallelgroup randomised controlled trial comparing a routine package of diagnostic tools (smear microscopy plus culture) with a novel one (sputum Xpert MTB/RIF (Cepheid, USA), urine lipoarabinomannan and culture) for ICF, which is reported elsewhere. The incidence of smear positivity and the potential implications for infectiousness and community-based transmission of TB are described here.

\section{Methods}

Study population

The study was conducted in two southern African communities: the Langa informal settlement, in Cape Town, SA, and the Mabvuku suburb, in Harare, Zimbabwe. Both have high TB and HIV prevalence, a high density of informal dwellings and high unemployment rates. The weekly screening locations were chosen in advance and were systematically clustered around community congregate settings or suspected transmission 'hot-spots' such as shops, hostels or transit hubs. The study vehicle was parked at these locations, and passersby were encouraged to participate via advertising banners displayed next to the vehicle, and by local advertising at schools, churches, supermarkets and social clubs. In 
SA, the vehicle was equipped with an awning for shelter, fold-up tables and capability for rapid HIV testing (Determine HIV-1/2, Alere, USA; HIV 1/2 3.0, Standard Diagnostics, South Korea) and point-of-care CD4count testing (PIMA, Alere, USA). The vehicle also included facilities to securely house and operate a GeneXpert machine (Cepheid, USA) and a small portable collapsible tent for sputum and urine acquisition. For patients unable to spontaneously produce sufficient sputum, facilities for sputum induction with an ultrasonic nebuliser and hypertonic saline using a standard protocol ${ }^{[13]}$ were also available in the tent. In Zimbabwe, a similar set-up was used for ICF but eligible participants were driven to the local Mabvuku community clinic where the same diagnostic tests were performed by a qualified technologist. Ethical approval to conduct the study was obtained from the relevant institutional review boards of the University of Cape Town and the University of Zimbabwe.

\section{Study procedures}

After obtaining informed consent, participants were counselled and their HIV status confirmed by fingerprick tests using two commercially available assays. The screening protocol asked about cough of any duration, haemoptysis, weight loss, fever and night sweats, according to a World Health Organization (WHO)-recommended screening algorithm, ${ }^{[2]}$ and enrollment depended on HIV status. We consecutively enrolled all consenting HIV-uninfected patients aged 18 years or older with at least one symptom of TB, but enrolled all consenting adult HIVinfected patients regardless of symptoms. The rationale for this latter strategy was that the 'rule-out' utility of the WHO screening algorithm is suboptimal in HIV-infected persons. ${ }^{[14]}$ Patients who refused HIV testing were screened as though they were HIV-infected. We excluded all patients who had previously self-presented to a TB community clinic in the last 2 months, had received treatment in the last 60 days, or who were unwilling or unable to give informed consent. At least two spot sputa were then obtained sequentially from each participant at recruitment, either spontaneously or via sputum induction. One specimen, selected arbitrarily, was sent to a reference laboratory for liquid culture for $M$. tuberculosis using the BACTEC MGIT 960 system (BD, USA). ${ }^{[15]}$ The other was used for either smear microscopy or Xpert MTB/RIF, according to assignment in the parent study. If possible, additional sputum specimens were collected for later analysis. We tested stored sputa of culture-positive patients for smear positivity in patients randomised to Xpert MTB/RIF in the parent study, at the end of the study.

\section{Statistical analysis}

Sputum culture positivity for M. tuberculosis complex was the diagnostic reference standard. Participants with contaminated cultures were excluded. We used $\chi^{2}$ and Fisher's exact test for comparisons between proportions. The Wilcoxon rank-sum test was used to compare non-parametric measures e.g. time to culture positivity. We also conducted a multivariable regression analysis of predictors of culture positivity, smear positivity and smear grade.

\section{Results}

\section{Study population}

Between October 2013 and April 2015, 2261 people were screened and 875 patients with suspected pulmonary TB were enrolled. A total of 646 had a valid smear microscopy result (Fig. 1). Overall, 16 were excluded from the primary analysis, of whom 13 had contaminated cultures and 3 were unable to produce additional sputum samples for culture.
Characteristics of culture-positive patients

Fifty-three participants (8.4\%) had a positive sputum culture for $M$. tuberculosis. The median (interquartile range (IQR)) age in patients with culture-positive TB was slightly lower (36 (29 - 42) v. 39 (31 - 48) years; $p=0.0377)$, and the median CD4 count significantly lower (185 (79 - 363) v. $306(163-515)$ cells $\left./ \mathrm{mm}^{3} ; p=0.0033\right)$, than in those participants who were culture negative (Table 1 ). The median number of WHO screening algorithm symptoms observed per patient with culture-positive TB was $3(2-4)$. The association between the duration of each symptom and either culture or smear positivity was not explored as this information was not recorded. Despite the screening protocol including all HIV-infected patients regardless of symptoms, $<1 \%$ of HIV-positive participants were completely asymptomatic and none of the culture-positive patients were asymptomatic. There was also no difference in the median number of symptoms in patients with and without TB $(3(2-4)$ v. $3(2-4) ; p=0.1946)$. The most frequent symptom was cough of more than 2 weeks, which had the highest sensitivity of the WHO symptoms for a positive TB culture but the lowest specificity ( $95.9 \%$ and $4.6 \%$, respectively; data reported in parent study). Each symptom either alone or in combination had poor discriminant ability for culture-positive disease.

\section{Characteristics of smear-positive patients}

The characteristics of smear-positive and smear-negative patients are shown in Table 2 . The only significant difference was that night sweats were more common in the smear-negative patients (97 v. $68 \%, p=0.004)$. Unsurprisingly, smear-positive patients had higher mycobacterial load, as measured by a shorter median culture time (in days) to positivity $(8(2-11)$ v. $24(15-30) ; p<0.0001)$.

\section{Diagnostic accuracy}

Smear microscopy detected 22/53 (42\%) of the culture-positive patients. The specificity, positive predictive value (PPV) and negative predictive value (NPV) for smear microscopy were 98.8, 75.9 and $94.8 \%$ overall (Table 3 ). There was no difference in sensitivity of smear microscopy in HIV-infected v. HIV-uninfected participants (A\% (95\% CI $22.9-57.7 \%)$ v. $45.0 \%$, (23.1 - 68.5\%); $p=0.06881)$.

Predictors of smear positivity and smear grade In a multivariable analysis, no clinical or demographic variable predicted smear positivity. Only decreased time-to-positivity was associated with smear grade (odds ratio (OR) 0.93, 95\% CI 0.91 - 0.96; $p<0.001$ ) (Fig. 2). Each symptom either alone or in combination had poor discriminant ability for smear-positive disease (Table 4). A symptom screen positive for all four WHO TB symptoms had the highest area under receiver operator curve (AUROC) characteristics, but a very low sensitivity (0.55, 95\% CI 0.46 - 0.64; 37.9\%, 95\% CI 20.7 - 57.7\%, respectively).

\section{Discussion}

We conducted a study to investigate novel strategies for detecting TB using community-based ICF in which we were able to study smearpositive (and thus potentially infectious) cases among participants with culture-proven TB. Our key findings were: $(i)$ a considerable proportion of patients with undiagnosed TB in the community (42\%) were smear positive, and hence potentially infectious; (ii) despite previous reports from primary care clinics suggesting a significant rate of subclinical disease, all cases of HIV-associated TB were symptomatic; (iii) no 


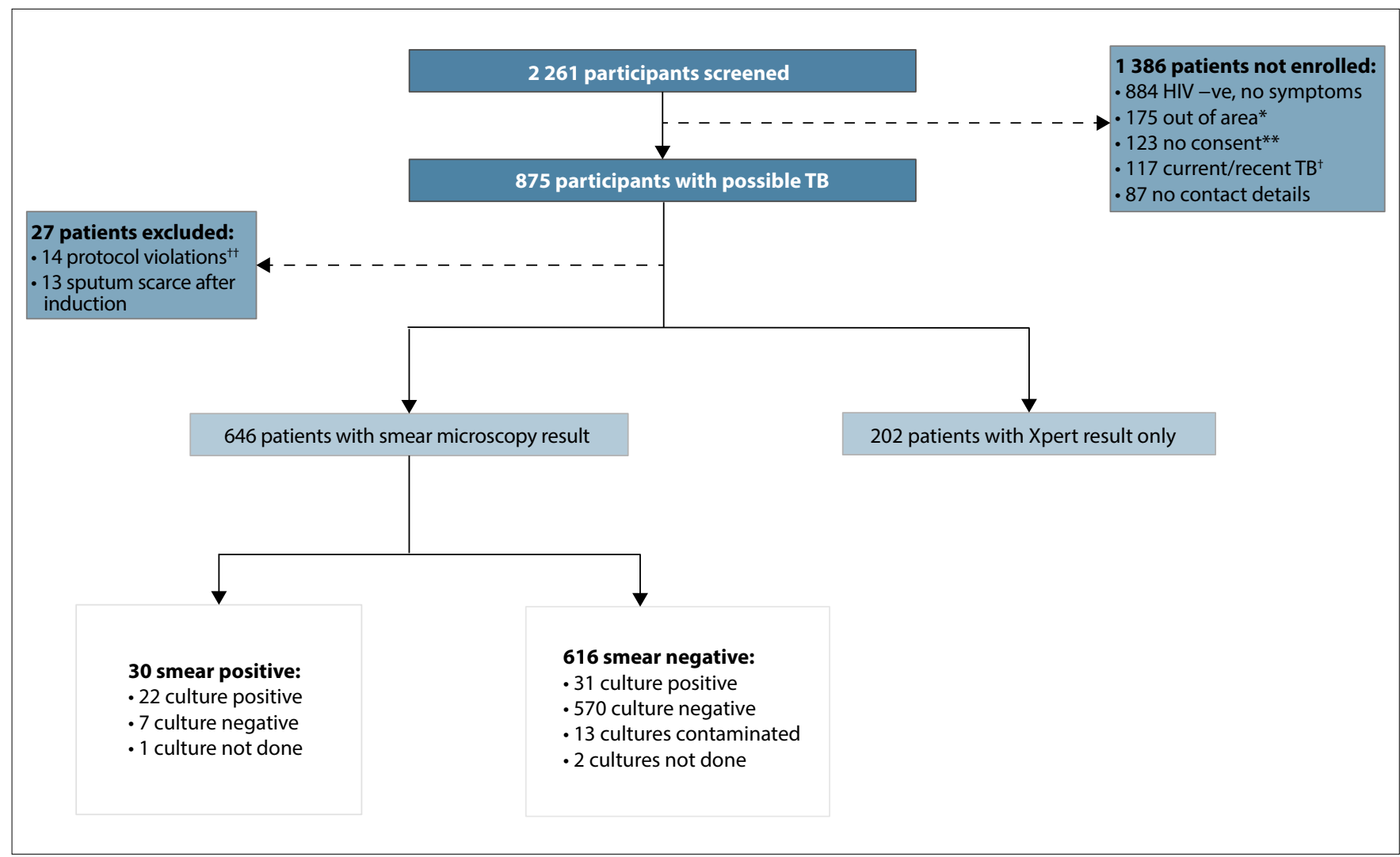

Fig. 1. CONSORT study diagram. ${ }^{\star}$ Patients temporarily visiting area of screening site but living elsewhere in the country and therefore unable to be followed up. ${ }^{*}$ Patients unable to consent (impaired/underage) or withdrawing consent. ${ }^{+}$Patients previously self-presenting to a TB community clinic in the last 60 days, or who had received treatment in the last 60 days. ${ }^{i t}$ Received test from the wrong diagnostics arm in error.

Table 1. Baseline characteristics of all patients receiving smear microscopy

\begin{tabular}{|c|c|c|c|c|}
\hline & Total $(N=646)$ & $\begin{array}{l}\text { Culture negative } \\
(n=557)\end{array}$ & $\begin{array}{l}\text { Culture positive } \\
(n=53)\end{array}$ & $p$-value \\
\hline \multicolumn{5}{|l|}{ Demographics } \\
\hline Age (years), median (IQR) & $39(31-48)$ & $39(31-48)$ & $36(29-42)$ & 0.0377 \\
\hline Males, $n(\%)$ & $285(44)$ & $249(43)$ & $26(49)$ & 0.4070 \\
\hline \multicolumn{5}{|l|}{ HIV indices } \\
\hline \multicolumn{5}{|l|}{ HIV status, $n(\%)$} \\
\hline Negative & $307(48)$ & $279(48)$ & $20(38)$ & 0.0856 \\
\hline Positive & $329(51)$ & $290(50)$ & $32(60)$ & 0.2467 \\
\hline Refused & $4(1)$ & $4(1)$ & $0(0)$ & 0.5359 \\
\hline Not done & $4(1)$ & $4(1)$ & $1(2)$ & 0.3672 \\
\hline CD4 count (cells $\left./ \mathrm{mm}^{3}\right)$, median (IQR) & $289(148-500)$ & $306(163-515)$ & $185(79-363)$ & 0.0033 \\
\hline ART use (if HIV +ve), $n(\%)$ & $144 / 376(38)$ & $130 / 334(39)$ & $11 / 34(32)$ & 0.5634 \\
\hline Duration of ART use (years), median (IQR) & $2.90(1.21-5.15)$ & $2.98(1.16-5.73)$ & $2.23(1.32-4.53)$ & 0.6149 \\
\hline \multicolumn{5}{|l|}{ Symptoms } \\
\hline Cough of any duration, $n(\%)$ & $608(94)$ & $545(95)$ & $50(94)$ & 0.9720 \\
\hline Weight loss, $n(\%)$ & $489(76)$ & $432(75)$ & $44(83)$ & 0.1860 \\
\hline Fever, $n(\%)$ & $282(44)$ & $254(44)$ & $23(43)$ & 0.9300 \\
\hline Night sweats, $n(\%)$ & $482(75)$ & $421(73)$ & $45(85)$ & 0.0580 \\
\hline Number of WHO screening symptoms, median (IQR) & $3(2-4)$ & $3(2-4)$ & $3(2-4)$ & 0.1936 \\
\hline
\end{tabular}


demographic variable (including HIV infection) or clinical symptom reliably identified participants at risk of smear-positive disease; and (iv) despite a clear relationship between smear grade and mycobacterial load

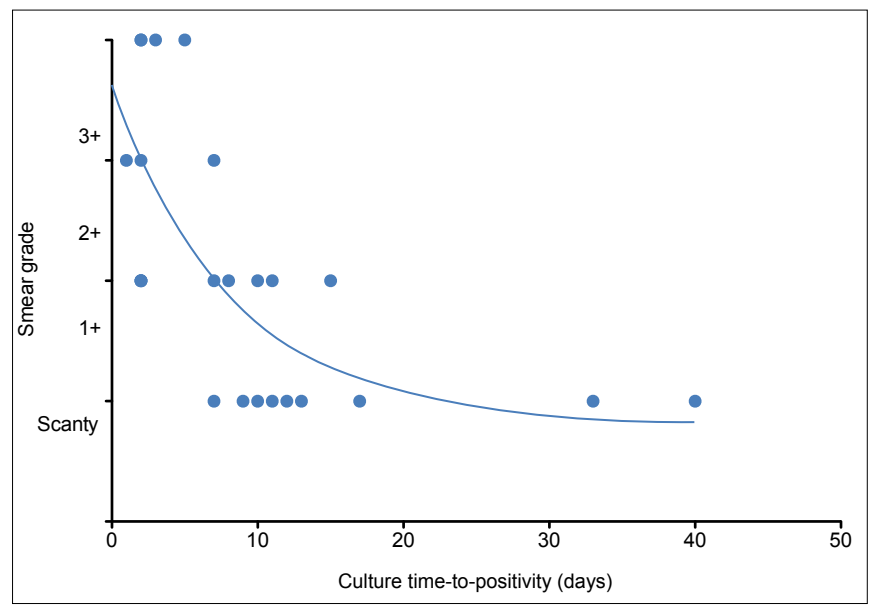

Fig. 2. Correlation with smear grade and culture time-to-positivity. (as measured by time-to-positivity), no demographic or clinical variable was associated with worsening smear grade.

Our study demonstrates the staggering burden of undiagnosed TB in the community in high-burden settings in sub-Saharan Africa. The prevalence of culture-positive TB among all participants presenting for screening was $3.27 \%$. This is considerably higher than the median of $0.8 \%$ reported from population-based surveys from sub-Saharan Africa. ${ }^{[16]}$ This may be explained by the high prevalence hotspots that were targeted in our study, with poor infrastructure and high rates of poverty, and the high prevalence of both HIV infection and symptoms of pulmonary tuberculosis in our participants. Furthermore, our findings are within the range of widely varying TB prevalence across eight studies (0.02 to 3.5\%) depending on the incidence of HIV, burden of TB, and whether all HIV-infected persons or only those with symptoms were screened.

The prevalence of smear-positive disease in our study was also high, approximating that seen in studies of symptomatic patients seeking care at primary health clinics for TB-related symptoms, ${ }^{[17]}$ or in patients investigated for TB symptoms in hospital. ${ }^{[18]}$ The consequences for

Table 2. Baseline characteristics of patients by smear status among TB cases

\begin{tabular}{|c|c|c|c|c|}
\hline & Total $(N=53)$ & $\begin{array}{l}\text { Smear negative } \\
(n=31)\end{array}$ & $\begin{array}{l}\text { Smear positive } \\
(n=22)\end{array}$ & $p$-value \\
\hline \multicolumn{5}{|l|}{ Demographics } \\
\hline Age (years), median (IQR) & $36(29-42)$ & $36(31-42)$ & $36(27-44)$ & 0.8637 \\
\hline Males, $n(\%)$ & $26(49)$ & $13(42)$ & $13(59)$ & 0.2180 \\
\hline \multicolumn{5}{|l|}{ HIV status, $n(\%)$} \\
\hline Negative & $20(38)$ & $11(35)$ & $9(41)$ & 0.6881 \\
\hline Positive & $32(60)$ & $20(66)$ & $12(55)$ & 0.4646 \\
\hline Refused & $1(2)$ & $0(0)$ & $1(5)$ & 0.2308 \\
\hline CD4 count, cells $/ \mathrm{mm}^{3}$, median (IQR) & $185(79-363)$ & $190(107-372)$ & $180(52-289)$ & 0.4910 \\
\hline ART use (if HIV +ve), $n(\%)$ & $11 / 32(34)$ & $8 / 20(40)$ & $3 / 12(25)$ & 0.3870 \\
\hline Duration of ART use (years), median (IQR) & $22.3(1.32-4.53)$ & $2.22(1.21-2.98)$ & $4.53(1.32-4.57)$ & 0.4142 \\
\hline \multicolumn{5}{|l|}{ Symptoms } \\
\hline Cough of any duration, $n(\%)$ & $50(94)$ & $28(90)$ & $22(100)$ & 0.1330 \\
\hline Weight loss, $n(\%)$ & $44(83)$ & $25(81)$ & $19(86)$ & 0.5850 \\
\hline Fever, $n(\%)$ & $23(43)$ & $11(35)$ & $12(55)$ & 0.1680 \\
\hline Night sweats, $n(\%)$ & $45(85)$ & $30(97)$ & $15(68)$ & 0.0040 \\
\hline Number of WHO screening symptoms, median (IQR) & $3(2-4)$ & $3(2-4)$ & $3(2-4)$ & 0.7166 \\
\hline \multicolumn{5}{|l|}{ Measures of infectivity } \\
\hline Time-to-positivity (days), median (IQR) & $15(8-28)$ & $24(15-30)$ & $8(2-11)$ & $<0.0001$ \\
\hline
\end{tabular}

Table 3. Diagnostic accuracy of smear microscopy, paired culture positive (excluding contaminated/unpaired cultures or cultures not done)

\begin{tabular}{lllll}
\hline & \multicolumn{4}{c}{ Test performance, $\boldsymbol{n}(\%, \mathbf{9 5 \%}$ CI $)$} \\
\cline { 2 - 5 } & Sensitivity & Specificity & PPV & NPV \\
\hline All patients & $22 / 53(41.5,28.1-55.9)$ & $570 / 577(98.8,97.5-99.5)$ & $22 / 29(75.9,56.5-89.7)$ & $570 / 601(94.8,92.8-96.5)$ \\
HIV uninfected & $9 / 20(45.0,23.1-68.5)$ & $281 / 283(99.3,97.5-99.9)$ & $9 / 11(81.8,48.2-97.7)$ & $281 / 292(96.2,93.4-98.1)$ \\
HIV infected & $13 / 33(39.4,22.9-57.7)$ & $289 / 294(99.3,97.5-99.9)$ & $13 / 18(72.2,46.5-90.3)$ & $209 / 221(93.5,90.2-96.0)$
\end{tabular}


transmission dynamics in these high burden settings is considerable: it is estimated that an infectious, smear-positive case is responsible for 15 - 20 transmission events per year ${ }^{[19,20]}$ and a strategy that could identify and treat these individuals in 28 days or less could be estimated to avert 1 - 2 transmission events per infectious case. The importance of ICF as a strategy for TB control in these communities is underscored.

Although we have focused on smear-positive disease, smear-negative cases, although less infectious than their smear-positive counterparts, are still responsible for between $17-20 \%$ of all TB transmission, ${ }^{[21,22]}$ and these patients comprised $58 \%$ of our cohort. Smear-negative cases (if undetected) eventually become smear-positive ones, and any ICF strategy should consider novel tools (such as Xpert) that allow early detection, as this is likely to have important downstream effects on transmission too.

The WHO advocates a simple 'rule-out' screening algorithm for TB in resource-limited settings whereby the absence of cough, fever, weight loss and night sweats portends a low probability of $\mathrm{TB}$, but the sensitivity of this approach has been shown to be suboptimal in HIV-infected individuals. ${ }^{[14]}$ For example, a study from Durban, SA, found that up to $22 \%$ of patients screened with sputum cultures prior to entering an ART programme had asymptomatic or subclinical TB. ${ }^{[23]}$ However, despite modifying our screening protocol to include all HIV-infected patients regardless of symptoms, we did not find any subclinical or asymptomatic TB among HIV-infected patients participating in our study. This finding is likely to be influenced in part by a selection bias, as HIV-positive individuals in the community were not screened in a systematic way. Overall, participants identified by this study were highly symptomatic, with a median number of three WHO symptoms reported. The symptom screen had poor discriminant ability for both TB disease and smear positivity. The social, logistical and behavioural reasons for why these highly symptomatic individuals do not access healthcare or do so late in the course of the disease, despite the local availability of TB and HIV services, require urgent further study, and would seem to contradict the findings of a previous case-finding study, also attached to a mobile testing service and conducted in Cape Town, which found that $44 \%$ of HIV-infected patients were asymptomatic..$^{[12]}$

There were several limitations to our study. Firstly, although we focused on smear positivity, there were several other unmeasured patient factors that may impact infectiousness. Recent cough aerosol experiments have shown that the characteristics of the bioaerosol produced in patients with pulmonary TB (particle quantity and size) may be just as important as measures of mycobacterial load as smear grade or culture time-topositivity. ${ }^{[24]}$ The collection of cough aerosol was beyond the scope of the study. We also did not perform chest X-rays as part of our ICF strategy. Cavitary disease, smear positivity and bacillary burden are known to be highly correlated, ${ }^{[25-27]}$ and radiological features may have outperformed clinical predictors of smear positivity. However, chest radiology was not included in our ICF algorithm in the parent study, by design: the necessity of transporting patients to an additional healthcare facility would have diluted the value of point-of-contact diagnosis, and would have affected both the cost and complexity of the ICF strategy. In addition, we only collected a single sputum specimen for smear microscopy on each patient. The collection of additional specimens is associated with incremental yield, and so the true proportion of smear-positive cases in this study has almost certainly been underestimated. Lastly, extrapulmonary samples were not routinely collected, so the number of TB cases in this study may also be an underestimate. However, this is less relevant to this study, as patients with extrapulmonary $\mathrm{TB}$ are less likely to contribute to the infectious pool targeted by ICF.

\section{Conclusion}

Almost half of patients with TB in the community detected by an ICF strategy were smear positive and hence potentially infectious. Neither HIV status nor symptoms identified those patients who were smear positive despite them having a higher mycobacterial burden. Further research is required to understand the epidemiology, behaviour and biology of symptomatic patients who fail to seek healthcare, or who do so late in the course of their disease, and what factors might identify potentially infectious patients so that limited resources can be appropriately targeted.

Role of the funding source. The funders of the study had no role in study design, data collection, data analysis, data interpretation or writing of the report. The corresponding author had full access to all the data in the study and had final responsibility for the decision to submit for publication.

Table 4. Diagnostic accuracy of WHO symptom screen for smear-positive TB

\begin{tabular}{|c|c|c|c|c|c|}
\hline & \multicolumn{4}{|c|}{ Test performance, $n(\%, 95 \% \mathrm{CI})$} & \multirow[b]{2}{*}{ AUROC } \\
\hline & Sensitivity & Specificity & PPV & NPV & \\
\hline \multirow[t]{2}{*}{ Cough of any duration } & $28 / 29$ & $34 / 601$ & $28 / 595$ & $34 / 35$ & 0.51 \\
\hline & $(96.6,82.2$ - 99.9) & $(5.7,3.8-7.8)$ & $(4.7,3.1-6.7)$ & $(97.1,85.1-99.9)$ & $(0.48-0.55)$ \\
\hline \multirow[t]{2}{*}{ Weight loss } & $26 / 29$ & $151 / 601$ & $26 / 476$ & $151 / 154$ & 0.57 \\
\hline & $(89.7,72.6-97.8)$ & $(25.1,21.7-28.8)$ & $(5.5,3.6-7.9)$ & $(98.1,94.4-99.6)$ & $(0.51-0.63)$ \\
\hline \multirow[t]{2}{*}{ Fever } & $14 / 29$ & $338 / 601$ & $14 / 277$ & $393 / 429$ & 0.52 \\
\hline & $(48.3,29.4-67.5)$ & $(56.2,52.2-60.2)$ & $(5.1,2.8-8.3)$ & $(95.8,93.1-97.6)$ & $(0.43-0.62)$ \\
\hline \multirow[t]{2}{*}{ Night sweats } & $18 / 29$ & $153 / 601$ & $18 / 466$ & $153 / 164$ & 0.44 \\
\hline & $(62.1,42.3-79.3)$ & $(25.5,22.0-29.1)$ & $(3.9,2.3-6.0)$ & $(93.3,88.3-96.6)$ & $(0.35-0.53)$ \\
\hline \multirow[t]{2}{*}{ Night sweats plus weight loss } & $17 / 29$ & $242 / 601$ & $17 / 376$ & $242 / 254$ & 0.49 \\
\hline & $(58.6,38.9-76.5)$ & $(40.3,36.3-44.3)$ & $(4.5,2.7-7.1)$ & $(95.3,91.9-97.5)$ & $(0.40-0.59)$ \\
\hline \multirow[t]{2}{*}{ All four WHO screening symptoms } & $11 / 29$ & $430 / 601$ & $11 / 171$ & $430 / 448$ & 0.55 \\
\hline & $(37.8,20.7-57.7)$ & $(71.5,67.8-75.1)$ & $(6.0,3.1-10.6)$ & $(96.0,93.7-97.6)$ & $(0.46-0.64)$ \\
\hline
\end{tabular}


1. Dheda K, Barry CE 3rd, Maartens G. Tuberculosis. Lancet 2016;387(10024):1211-1226. http://dx.doi.org/10.1016/S0140-6736(15)00151-8

2. World Health Organization. Systematic screening for active tuberculosis: Principles and recommendations. 2013. http://www.who.int/tb/publications/Final_TB_Screening guidelines.pdf (accessed 1 April 2015).

3. Glynn JR, Guerra-Assuncao JA, Houben RM, et al. Whole genome sequencing shows a low proportion of tuberculosis disease is attributable to known close contacts in rural Malawi. PLoS One 2015;10(7):e0132840. http://dx.doi.org/10.1371/journal.pone.0132840

4. Middelkoop K, Mathema B, Myer L, et al. Transmission of tuberculosis in a South African community with a high prevalence of HIV infection. J Infect Dis 2015;211(1):53-61. http:// dx.doi.org/10.1093/infdis/jiu403

5. Verver S, Warren RM, Munch Z, et al. Proportion of tuberculosis transmission that takes place in households in a high-incidence area. Lancet 2004;363(9404):212-214. http:// dx.doi.org/10.1016/S0140-6736(03)15332-9

6. Loudon RG, Williamson J, Johnson JM. An analysis of 3485 tuberculosis contacts in the city of Edinburgh during 1954-1955. Am Rev Tuberc 1958;77(4):623-643.

7. Sepkowitz KA. How contagious is tuberculosis? Clin Infect Dis 1996;23(5):954-962. http:// dx.doi.org/10.1093/clinids/23.5.954

8. Shaw JB, Wynn-Williams N. Infectivity of pulmonary tuberculosis in relation to sputum status. Am Rev Tuberc 1954;69(5):724-732.

9. Grzybowski S, Barnett GD, Styblo K. Contacts of cases of active pulmonary tuberculosis. Bull Int Union Tuberc 1975;50(1):90-106.

10. Riley RL, Moodie AS. Infectivity of patients with pulmonary tuberculosis in inner city homes. Am Rev Respir Dis 1974;110(6):810-812. http://dx.doi.org/10.1164/arrd.1974.110.6P1.810

11. Yeager H, Jr, Lacy J, Smith LR, LeMaistre CA. Quantitative studies of mycobacterial populations in sputum and saliva. Am Rev Respir Dis 1967;95(6):998-1004. http://dx.doi. org/10.1164/arrd.1967.95.6.998

12. Kranzer K, Lawn SD, Meyer-Rath G, et al. Feasibility, yield, and cost of active tuberculosis case finding linked to a mobile HIV service in Cape Town, South Africa: A cross-sectional study. PLoS Med 2012;9(8):e1001281. http://dx.doi.org/10.1371/journal.pmed.1001281

13. Peter JG, Theron G, Singh N, Singh A, Dheda K. Sputum induction to aid diagnosis of smear-negative or sputum-scarce tuberculosis in adults in HIV-endemic settings. Eur Respir J 2014;43(1):185-194. http://dx.doi.org/10.1183/09031936.00198012

14. Getahun H, Kittikraisak W, Heilig CM, et al. Development of a standardized screening rule for tuberculosis in people living with HIV in resource-constrained settings: Individual participant data meta-analysis of observational studies. PLoS Med 2011;8(1):e1000391. http://dx.doi.org/10.1371/journal.pmed.1000391

15. Cruciani M, Scarparo C, Malena M, Bosco O, Serpelloni G, Mengoli C. Meta-analysis of BACTEC MGIT 960 and BACTEC 460 TB, with or without solid media, for detection of mycobacteria. J Clin Microbiol 2004;42(5):2321-2325.
16. Kranzer K, Houben RM, Glynn JR, Bekker LG, Wood R, Lawn SD. Yield of HIVassociated tuberculosis during intensified case finding in resource-limited settings: A systematic review and meta-analysis. Lancet Infect Dis 2010;10(2):93-102. http:// dx.doi.org/10.1016/s1473-3099(09)70326-3

17. Theron G, Zijenah L, Chanda D, et al. Feasibility, accuracy, and clinical effect of point-of-care Xpert MTB/RIF testing for tuberculosis in primary-care settings in Africa: A multicentre, randomised, controlled trial. Lancet 2014;383(9915):424-435. http://dx.doi.org/10.1016/s0140-6736(13)62073-5

18. Theron G, Peter J, Zyl-Smit R, Mishra H, Streicher E, Murray S. Evaluation of the Xpert MTB/RIF assay for the diagnosis of pulmonary tuberculosis in a high HIV prevalence setting. Am J Respir Crit Care Med 2011;184(1):132-140. http://dx.doi. org/10.1164/rccm.201101-0056OC

19. Houben RM, Dowdy DW, Vassall A, et al. How can mathematical models advance tuberculosis control in high HIV prevalence settings? Int J Tuberc Lung Dis 2014;18(5):509-514. http://dx.doi.org/10.5588/ijtld.13.0773

20. Zwerling A, White RG, Vassall A, Cohen T, Dowdy DW, Houben RM. Modeling of novel diagnostic strategies for active tuberculosis - a systematic review: Current practices and recommendations. PLoS One 2014;9(10):e110558. http://dx.doi. org/10.1371/journal.pone. 0110558

21. Behr MA, Warren SA, Salamon H, et al. Transmission of Mycobacterium tuberculosis from patients smear-negative for acid-fast bacilli. Lancet 1999;353(9151):444-449. http://dx.doi.org/10.1016/S0140-6736(98)03406-0

22. Tostmann A, Kik SV, Kalisvaart NA, et al. Tuberculosis transmission by patients with smear-negative pulmonary tuberculosis in a large cohort in the Netherlands. Clin Infect Dis 2008;47(9):1135-1142. http://dx.doi.org/10.1086/591974

23. Bassett IV, Wang B, Chetty S, et al. Intensive tuberculosis screening for HIV-infected patients starting antiretroviral therapy in Durban, South Africa. Clin Infect Dis 2010;51(7):823-829. http://dx.doi.org/10.1086/656282

24. Fennelly KP, Jones-Lopez EC, Ayakaka I, et al. Variability of infectious aerosols produced during coughing by patients with pulmonary tuberculosis. Am J Respir Crit Care Med 2012;186(5):450-457. http://dx.doi.org/10.1164/rccm.2012030444OC

25. Lau A, Barrie J, Winter C, Elamy AH, Tyrrell G, Long R. Chest radiographic patterns and the transmission of tuberculosis: Implications for automated systems. PLoS One 2016;11(4):e0154032. http://dx.doi.org/0.1371/journal.pone.0154032

26. Lohmann EM, Koster BF, Le Cessie S, Kamst-van Agterveld MP, van Soolingen $\mathrm{D}$, Arend SM. Grading of a positive sputum smear and the risk of Mycobacterium tuberculosis transmission. Int J Tuberc Lung Dis 2012;16(11):1477-1484. http:// dx.doi.org/10.5588/ijtld.12.0129

27. Palaci M, Dietze R, Hadad DJ, et al. Cavitary disease and quantitative sputum bacillary load in cases of pulmonary tuberculosis. J Clin Microbiol 2007;45(12):40644066. http://dx.doi.org/10.1128/JCM.01780-07 